\title{
Research Article Digital-Signal-Type Identification Using an Efficient Identifier
}

\author{
Ataollah Abrahamzadeh, ${ }^{1}$ Seyed Alireza Seyedin, ${ }^{2}$ and Mehdi Dehghan ${ }^{3}$ \\ ${ }^{1}$ Faculty of Electrical and Computer Engineering, Noshirvani Institute of Technology, Mazandauan University, \\ P.O. Box 47148-71167, Babol, Iran \\ ${ }^{2}$ Faculty of Electrical Engineering, Department of Electrical Engineering, Ferdowsi University of Mashad, \\ P.O. Box 91779-48974, Mashad, Iran \\ ${ }^{3}$ Faculty of Computer Engineering and Information Technology, Amirkabir University of Technology, P.O. Box 15914, \\ Tehran, Iran
}

Received 14 September 2006; Revised 10 February 2007; Accepted 4 April 2007

Recommended by Enis Ahmet Cetin

\begin{abstract}
Automatic digital-signal-type identification plays an important role for various applications. This paper presents a highly efficient identifier (technique) that identifies a variety of digital signal types. In this technique, a selected number of the higher-order moments and the higher-order cumulants up to eighth are utilized as the effective features. A hierarchical support-vector-machine(SVMs) based structure is proposed for multiclass classification. A genetic algorithm is proposed in order to improve the performance of the identifier. Genetic algorithm selects the suitable parameters of SVMs that are used in the structure of the classifier. Simulation results show that the proposed identifier has high performance for identification of the considered digital signal types even at very low SNRs.
\end{abstract}

Copyright ( $\odot 2007$ Ataollah Abrahamzadeh et al. This is an open access article distributed under the Creative Commons Attribution License, which permits unrestricted use, distribution, and reproduction in any medium, provided the original work is properly cited.

\section{INTRODUCTION}

Automaticdigital-signal-type identification is a technique that recognizesthe type ofreceived signal and plays an important role in various applications. For example, in military applications, it can be employed for electronic surveillance and monitoring; in civil applications, it can be used for spectrum management, network traffic administration, signal confirmation, interference identification, software radios, multidrop networks, intelligent modems, and so forth.

In the past, signal-type identification relied mostly on operators scanning the radio frequency spectrum with a wide-band receiver and checking it visually on some sort of display [1]. Clearly, these methods relied very much on the operators' skills and abilities. These limitations then led to the development of more automated modulation recognizers. One semiautomatic approach was to run the received signal through a number of demodulators and then have an operator to determine the modulation format by listening to the output of each demodulator. This approach is however not very practical anymore due to the new digital techniques that transfer both voice and data. Then techniques for automatic signal-type identification started to emerge. The re- cent contributions in the subject focus more on the digital signal types, specially the higher-order digital signals, due to increasing usage of such types in many novel applications.

Automatic digital-signal-type identification techniques usually can be categorized in two main principles: the decision-theoretic (DT) and the pattern recognition (PR). DT techniques use probabilistic and hypothesis testing arguments to formulate the recognition problem $[2,3]$. The decision-theoretic (DT) techniques have many drawbacks. These techniques are not robust with respect to model mismatch [4]. Another problem is the high computational complexity [4]. Other problems are difficulties in forming the right hypothesis testing as well as careful analysis that are required to set the correct threshold values [5]. However, PR techniques do not need such careful treatment [5]. They are easy to implement. PR techniques can be further divided into two main subsystems: the feature extraction and the classifier. The former extracts the features and the latter determines the membership of signal [4-17].

In [7], the authors proposed a technique for identification of ASK2, ASK4, PSK2, PSK4, FSK2, and FSK4 signals. The classifier is based on a decision flow. These digital signal types have been identified with a success rate around $90 \%$ at 
SNR $=10 \mathrm{~dB}$. In [4], the authors proposed a digital-signal type identification technique based on elementary fourthorder cumulant. When it was used for identification of the BPSK, PAM4, QAM16, and PSK8, the success rate was about $96 \%$ at $\mathrm{SNR}=10 \mathrm{~dB}$. In [8], the authors proposed a technique to discriminate among ASK, 4DPSK, 16QAM, and FSK digital signals. The chosen features are the kurtosis of the signal, the number of peaks in the phase probability density function (PDF), and the mean of the absolute value signal frequency. A fuzzy classifier was used in this technique. For SNR $>5 \mathrm{~dB}$, the identifier worked properly. When SNR was less than $5 \mathrm{~dB}$, the performance was worse. In [9], for the first time, Ghani and Lamontagne proposed using the multilayer perceptron (MLP) neural network with backpropagation (BP) learning algorithm for automatic signal-type identification. They showed that neural network classifier outperforms other classifiers such as K-nearest neighbor (KNN). In [10], the authors showed that the neural network classifier has a higher performance than the threshold classifier. In [11], the authors proposed an identifier for the identification of PSK2, PSK4, PSK8, OQPSK, MSK, QAM16, QAM64, FSK2, and FSK4 signal types. The features chosen to characterize the signal types are the mean and the next three moments of the instantaneous characteristics. They used different classifiers and showed that the artificial neural network has better performance than K-nearest neighbor (KNN) classifier and the well known binary decision trees. They reporteda success rate of $93 \%$ with SNR range $15-25 \mathrm{~dB}$. However, the performance for lower SNRs is reported to be less than $80 \%$. In [12], the authors proposed an identifier based on cyclic spectral features for identification of AM, USB, LSB, FM, ASK, FSK, BPSK, QPSK, and SQPSK. It was claimed that cyclic spectrum posses more advantage than power spectrum in signal-type recognition. A full-connected backpropagation neural network is used for classification in that research. The success rate of this identifier is reported around $90 \%$ with SNR range 5-25 dB. In [5], the authors have used a combination of spectral features and statistical features (second, third, and fourth orders of cumulants) for identification of ASK2, ASK4, PSK2, PSK4, FSK2, FSK4, V29, V32, QAM16, and QAM64. The classifier was an MLP neural network. They reported a high success rate at most of SNRs. The authors did not clarify the correction percentage of each of the modulations individually. Also they have used a fully connected neural network. This causes a long training time as well as the high complexity of the classifier. If the number of samples reduces, the performance will drop. In [13], the authors have done a comparative study of implementation of feature extraction and classification algorithms based on discrete wavelet decompositions and adaptive-network based fuzzy interference System (ANFIS) for recognition of ASK8, FSK8, PSK8, and QASK8.

It can be found that the techniques that use MLP neural networks as the classifier have high performances. However, with regard to effectiveness of MLP neural networks, there are some problems. For example, MLP neural networks have limitations on generalization ability in low SNRs. Another main drawback of the MLP models is that the training proce- dure often gets stuck at a local optimum of the cost function [15].

In recent years, support vector machines (SVMs), based on statistical learning theory, are gaining applications in area of pattern recognition and detection of microcalcifications in digital mammograms, because of excellent generalization capability [18]. In [14], the authors proposed an identifier for signal-type identification that uses a binary SVM as the classifier. The features were extracted using wavelet packet analysis and biorthogonal wavelet. The accuracy of the proposed identifier exceeds $98 \%$ for SNR > $4 \mathrm{~dB}$. In [15], Wu et al. introduced an identifier for automatic digital modulation recognition method based on SVMs. They have used the five key features that are introduced in [7]. It is shown that this method can achieve a satisfying performance at an SNR as low as $5 \mathrm{~dB}$. This algorithm can recognize the modulation types of ASK2, ASK4, FSK2, FSK4, PSK2, and PSK2. As mentioned in [5], these features are only suitable for these low orders of signals that contain hidden information only in instantaneous amplitude, the instantaneous phase, and/or the instantaneous frequency. Using the higherorder statistics makes higher performances for fault detection systems $[18,19]$. In [16], the authors proposed four features to classify ASK2, ASK4, PSK2, PSK4, FSK2, and FSK4. The features were extracted based on two main processing steps. The first step is the multiplication of two consecutive signal values. In the second step, the mean, the kurtosis of real and imaginary parts of the quantity obtained in the first step were used as the input features of the SVMs. In [17], Gang et al. proposed an identifier for recognition of ASK4, PSK2, PSK4, PSK8, and QAM16. The probability of correct classification was about $98 \%$ at an SNR of $4 \mathrm{~dB}$.

From the published works, it can be found that the identifiers, which use the statistical features, are able to include the digital signal types such as QAM and higher orders of digital signals. Also, the techniques that use SVMs as the classifier have high performances at low-level SNRs. In this paper, we propose a highly efficient identifier which contains the mentioned specifications. It uses a selected combination of the higher-order moments and the higher-order cumulants (up to eighth) as the effective features for representation of digital signals. We have proposed a new and simple multiclass SVM-based classifier that has a hierarchical structure. Suitable parameters of SVMs can improve the performance of the identifier. We have proposed a genetic algorithm (GA) for tuning the parameters of SVMs that are used in the proposed classifier.

Figure 1 shows the general scheme of the proposed identifier. The preprocessing module performs actions such as rejection of noise outside of the signal bandwidth, carrier frequency estimation (or to be known), recovery of complex envelope. This stage is similar in most of the techniques, and hence we will not explain it more. The feature extraction module is presented in Section 2. Also the digital signaltypes set (DSTS) that is considered in this paper is introduced in Section 2. The classifier module is described in Section 3. Optimization problem using GA is presented in Section 4. 


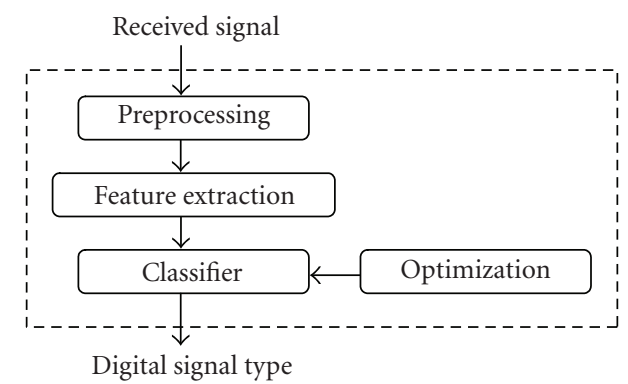

Figure 1: General scheme of the proposed identifier.

Section 5 shows some simulation results. Finally, Section 6 concludes the paper.

\section{DSTS AND FEATURE EXTRACTION}

Different types of digital signal have different characteristics. Therefore finding the proper features for the recognition of digital signals, particularly in case of higher-order and/or nonsquare kinds of digital signal, is a serious problem. In this paper, DSTS is ASK4, ASK8, PSK2, PSK4, PSK8, Star-QAM8, V29, QAM32, and QAM64. Because of simplifying the indication, the digital signal types of ASK4, ASK8, PSK2, PSK4, PSK8, Star-QAM8, V29, QAM32, and QAM64 are substituted with $\mathrm{P}_{1}, \mathrm{P}_{2}, \mathrm{P}_{3}, \mathrm{P}_{4}, \mathrm{P}_{5}, \mathrm{P}_{6}, \mathrm{P}_{7}, \mathrm{P}_{8}$, and $\mathrm{P}_{9}$, respectively. Among the different features that we have computed and experimented, the higher-order moments and higher-order cumulants (up to eighth) make the highest performances for identification of DSTS. These features can provide a fine way to describe the shape of the probability density function. The following subsections briefly describe these features.

\subsection{Moments}

Probability distribution moments are a generalization of the concept of the expected value. Recall that the general expression for the $i$ th moment of a random variable is given by [20]

$$
\mu_{i}=\int_{-\infty}^{\infty}(s-m)^{i} f(s) d s,
$$

where $m$ is the mean of the random variable. The definition for the $i$ th moment for a finite length discrete signal is given by

$$
\mu_{i}=\sum_{k=1}^{N}\left(s_{k}-\mu\right)^{i} f\left(s_{k}\right),
$$

where $N$ is the data length. In this study, signals are assumed to be zero mean. Thus,

$$
\mu_{i}=\sum_{k=1}^{N} s_{k}^{i} f\left(s_{k}\right) .
$$

Next, the automoment of the random variable may be defined as follows:

$$
M_{p q}=E\left[s^{p-q}\left(s^{*}\right)^{q}\right]
$$

TABLE 1: Some of the features for a number of digital signal types.

\begin{tabular}{lccccc}
\hline & $\mathrm{P}_{1}$ & $\mathrm{P}_{3}$ & $\mathrm{P}_{4}$ & $\mathrm{P}_{6}$ & $\mathrm{P}_{9}$ \\
\hline$M_{41}$ & 1.64 & 1 & 0 & 0 & 0 \\
$M_{61}$ & 2.92 & 1 & -1 & 2.92 & -1.3 \\
$C_{63}$ & 8.32 & 16 & 4 & .160 & 1.79 \\
$M_{84}$ & 5.24 & 1 & 1 & 5.25 & 3.96 \\
$C_{80}$ & -30.1 & -244 & 34 & -88.9 & -11.5 \\
$C_{82}$ & -30.1 & -244 & -46 & 63.31 & -27.1 \\
\hline
\end{tabular}

where $p$ is called the moment order and $s^{*}$ stands for complex conjugation of $s$.

Assume a zero-mean discrete basedband signal sequence of the form $s_{k}=a_{k}+j b_{k}$. Using the definition of the automoments, the expressions for different orders may be easily derived. For example,

$$
M_{41}=E\left[(a+j b)^{3}(a-j b)\right]=E\left[a^{4}-b^{4}\right] .
$$

\subsection{Cumulants}

Consider a scalar zero-mean random variable $s$ with characteristic function:

$$
\widehat{f}(t)=E\left\{e^{j t s}\right\}
$$

Expanding the logarithm of the characteristic function as a Taylor series, one obtains

$$
\log \hat{f}(t)=k_{1}(j t)+\cdots+\frac{k_{r}(j t)^{r}}{r !}+\cdots .
$$

The constants $k_{r}$ in (7) are called the cumulants (of the distribution) of $s$. The symbolism for $p$ th order of cumulant is similar to that of the $p$ th-order moment. More specially,

$$
C_{p q}=\operatorname{Cum}[\underbrace{s, \ldots, s}_{(p-q) \text { terms }}, \underbrace{s^{*}, \ldots, s^{*}}_{(q) \text { terms }}] .
$$

For example:

$$
C_{81}=\operatorname{Cum}\left(s, s, s, s, s, s, s, s^{*}\right) .
$$

We have computed all of the features for DSTS. Table 1 shows some of these features for a number of the considered digital signal types. These values are computed under the constraints of unit variance and noise free.

\section{CLASSIFIER}

We have proposed a multiclass SVM-based classifier that has a hierarchical structure. SVMs were introduced on the foundation of statistical learning theory. The basic SVM deals with two-class problems; however, it can be developed by some special methods for multiclass classification [21]. Binary SVM performs classification tasks by constructing the optimal separating hyperplane $(\mathrm{OSH})$. OSH maximizes the margin between the two nearest data points belonging to the two separate classes. 
Suppose that thetraining set $\left(x_{i}, y_{i}\right), i=1,2, \ldots, l, x \in$ $\mathbb{R}^{d}, y \in\{-1,+1\}$, can be separated by the hyperplane $w^{T} x+b=0$, where $w$ is the weight vector and $b$ is the bias. If this hyperplane maximizes the margin, then the following inequality is valid for all input data:

$$
y_{i}\left(\mathbf{w}^{T} \mathbf{x}_{i}+b\right) \geq 1, \quad \forall \mathbf{x}_{i}, i=1,2, \ldots, l .
$$

Those training points, for which the equality in (10) holds, are called support vectors (SVs). The margin of the hyperplane is equal to $2 /\|w\|$. Thus, the problem is the maximizing of the margin by minimizing of $\|w\|^{2}$ subject to (10). This is a convex quadratic programming (QP) problem. Lagrange multipliers $\left(\alpha_{i}, i=1, \ldots, l ; \alpha_{i} \geq 0\right)$ are used to solve it. Having done some computations, the optimal values $w$ and $b$ are achieved. Then the optimal decision function (ODF) is then given [21]:

$$
f(\mathbf{x})=\operatorname{sgn}\left(\sum_{i=1}^{l} y_{i} \alpha_{i}^{*} \mathbf{x}^{T} \mathbf{x}_{i}+b^{*}\right),
$$

where $\alpha_{i}^{*}$ 's are optimal Lagrange multipliers.

For inputs data with a high noise level, SVM uses soft margins that can be expressed as follows with the introduction of the nonnegative slack variables $\xi_{i}, i=1, \ldots, l$ :

$$
y_{i}\left(w^{T} x_{i}+b\right) \geq 1-\xi_{i} \quad \text { for } i=1,2, \ldots, l .
$$

To obtain the OSH, the $\Phi=(1 / 2)\|\mathbf{w}\|^{2}+C \sum_{i=1}^{l} \xi_{i}^{k}$ should be minimized subject to (12), where $C$ is the penalty parameter, which controls the tradeoff between the complexity of the decision function and the number of training examples, misclassified.

In the nonlinearly separable cases, the SVM map the training points, nonlinearly, to a high-dimensional feature space using kernel function $K\left(\vec{x}_{i}, \vec{x}_{j}\right)$, where linear separation may be possible. Gaussian radial basis function (GRBF) is one of the kernelfunctions. It is given by

$$
K(x, y)=\exp \left(\frac{-\|x-y\|^{2}}{2 \sigma^{2}}\right)
$$

where $\sigma$ is the width of the RBF kernel. After a kernel function is selected, the decision function will become

$$
f(\mathbf{x})=\operatorname{sgn}\left(\sum_{i=1}^{l} y_{i} \alpha_{i}^{*} K\left(\mathbf{x}, \mathbf{x}_{i}\right)+b^{*}\right) .
$$

The performance of an SVM depends on penalty parameter $(C)$ and the kernel parameter, which are called hyperparameters. In this paper, we have used the GRBF, because our extensive simulation shows that it has better performance than other kernels. Thus hyperparameters are $C$ and $\sigma$.

There are two widely used methods to extend binary SVMs to multiclass problems: one-against-all (OAA) method and one-against-one (OAO) method [22]. In this paper, we have proposed a hierarchical SVM-based classifier. Figure 2 shows the scheme of this classifier. One of the advantages of this structure is that the number of SVMs is less than in cases of OAO and OAA.

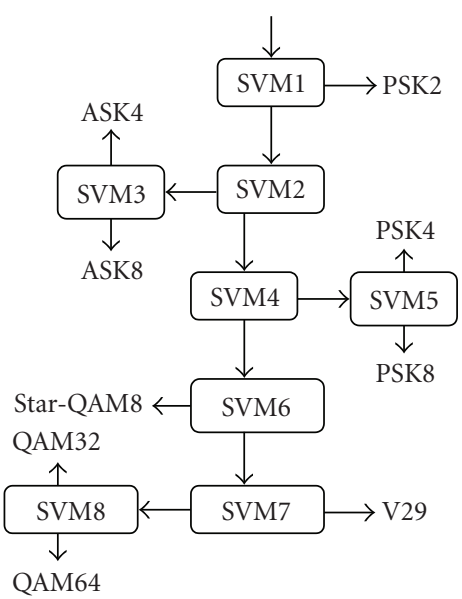

FIgURE 2: Hierarchical SVM-based classifier.

\section{GA FOR SELECTION OF THE PARAMETERS OF SVMS}

Finding the optimum values of the hyperparameters improves the performance of SVMs; however, it is a difficult problem [23]. GAs with their characteristics of high efficiency and global optimization are widely applied in many areas. In this paper, we have used GA for finding the optimum values of hyperparameters of SVMs. GA is a stochastic optimization algorithm, which adopts Darwin's theory of survival of the fittest. To apply a genetic algorithm, one has to define its basic issues.

Selection of the parameters of SVM is an optimization problem with constraints. Here, real-encoded scheme is selected as the representation of the parameters. The research space of these parameters is $C \in[2: 4: 50], \sigma \in[0.1: 0.1: 2]$. The size of the population (pop_size) is chosen to be 16 in order to avoid difficulties in the convergence of the population. For producing the initial population, the initial values of the designed parameters are distributed in the solution space as even as possible. According to the aforementioned analysis, the average performance of the SVM classifier is dependent on $E\left\{R^{2} / \gamma^{2}\right.$ ) and not simply on the large margin $\gamma$. The radius-margin bound is proposed as the fitness function [23]:

$$
T=\frac{1}{l} \frac{R^{2}}{\gamma^{2}}
$$

where $\gamma$ denotes the margin, $l$ is the size of the training samples, $R$ is the radius of the smallest sphere containing the training data, $R=0.5$.

Genetic operators include selection operator, crossover operator, and mutation operator. Here the method of survival of the fittest was used to select the next-generation individual. Given the fitness function fit $\left(a_{i}\right)$ of the individual $a_{i}$, the probability of $a_{i}$ selected as the next generation one is as follows:

$$
P\left(a_{i}\right)=\frac{\text { fit }\left(a_{i}\right)}{\sum_{j=1}^{\text {pop_size }} \operatorname{fit}\left(a_{j}\right)} \times \text { pop_size }
$$


TABLE 2: Chosen features for each SVM.

\begin{tabular}{cc}
\hline Number of SVMs & Chosen features \\
\hline SVM1 & $C_{81}$ \\
SVM2 & $M_{41}$ \\
SVM3 & $M_{41}, C_{81}$ \\
SVM4 & $M_{63}$ \\
SVM5 & $C_{61}$ \\
SVM6 & $C_{63}$ \\
SVM7 & $M_{82}, C_{80}$ \\
SVM8 & $M_{61}, C_{80}$ \\
\hline
\end{tabular}

The crossover operator is defined as [24]

$$
X^{\prime}=a X_{1}+(1-a) X_{2}
$$

where $X^{\prime}$ is the offspring aftercrossover operation, $X_{1}$ and $X_{2}$ are two parents to be implemented in the crossover operation, and $a$ is a constant which belongs to $(0,1)$. Here $a=0.5$. How the bigger value of the mutation operator is chosen to maintain the diversity of the population in the early GA operation and avoid the precocity? The adaptive mutation probability is adopted in this paper to solve the above two problems as follows:

$$
P_{m}=\frac{\exp (-b \times t / 2)}{\text { pop_size } \times \sqrt{L}},
$$

where $t$ is the generation of the genetic iteration, pop_size is the size of the population, $L$ is the length of the individual, $b=1.5$ is a preset parameter. In this paper, genetic algorithm terminates the program when the best fitness has not changed more than a very small value, that is, $10^{-6}$ over the last generations.

\section{SIMULATION RESULTS}

We have used Matlab environment for simulations. The simulated signals were band-limited and Gaussian noise was added according to SNR values -3, 0, 3, 6, 9, and $18 \mathrm{~dB}$. For each signal type, 1260 samples are used for simulations. Six hundred and thirty samples are used for training phase and 630 samples are used for testing phase. Among the features that we have mentioned in Section 2, Table 2 shows the chosen features that achieve the best results for identification of DSTS. These features were selected based on try and error.

\subsection{Performance without optimization}

Based on some experiments, the values $\sigma=1$ and $C=10$ are selected for all SVMs. Table 3 shows the diagonal matrix $(\mathrm{DM})$ or accuracy matrix (ACCM) at SNR $=3 \mathrm{~dB}$. Table 4 shows the identification results (performances) for DSTS in different SNR values. These are the averages of the values that appear in the diagonal of DM (or ACCM). It can be seen that the performance is generally very good even at very low SNRs. This is due to two facts: chosen features and novel classifier. The chosen features have the effective properties in signal representation. On the other hand, SVM-based classifier has high generalization ability for classification of the considered digital signals at low SNRs.

In order to compare the performance of the proposed hierarchical SVM-based classifier with another classifier, we have considered a hierarchical MLP-based classifier in which SVMs are replaced with MLP neural networks. These MLPs use backpropagation with momentum and adaptive learning rate algorithm. The simulation setups are the same. We name this technique as TECH2. Figure 3 shows the performances of two identifiers in different SNR values. It can be seen that the proposed technique (PROTECH) that uses SVM in the structure of its classifier has higher accuracy than TECH2, particularly, in low levels of SNR. When SNR is low, TECH2 shows poor performance, while in higher SNR the accuracy is higher. The construction of neural network in low SNRs is not proper, which results in low generalization ability. In higher SNRs, the features are proper and closer to the noiseless state and it is easier to construct the neural network and results in high identification probability.

In order to indicate the effectiveness of the chosen features, we have used the features that have been introduced in [4]. The structure of the classifier and the simulation setups are the same. We name this technique as TECH3. Figure 4 shows the performances of two identifiers. Results imply that our chosen features have highly effective properties in signal representation.

\subsection{Performance with applying $G A$}

In this section, we apply GA for finding the optimum parameters of SVMs that are in the structure of the proposed classifier. Table 5 shows the performances of the optimized identifier for various SNRs. Figure 5 shows a comparison between the performances of the nonoptimized technique (PROTECH) and optimized technique (OPROTECH). It can be seen that the optimization improves the performances of identifier for all SNRs, especially in lower SNRs. Table 6 shows the optimum parameters of SVMs that are used in the hierarchical structure. Table 7 indicates the diagonal matrix of identifier at $\mathrm{SNR}=3 \mathrm{~dB}$. Also, we have computed the performances of the optimized identifier at a high SNR value. Table 8 indicates the training performance of the identifier at $\mathrm{SNR}=40 \mathrm{~dB}$. It can be seen that the proposed identifier can show up to $100 \%$ accuracy.

\subsection{Performance comparison}

As mentioned in [5], direct comparison with other works is too difficult in signal-type identification. This is mainly because of the fact that there is no available single unified data set. Different setups of digital signal types will lead to different performances. Compared with other identifiers mentioned in Section 1, the proposed identifier in this paper has many advantages. This identifier has a simple structure and includes a variety of digital signal types. Each SVM in the identifier uses the features vector in order to map the input vectors' nonlinearity into high-dimensional feature space in a nonlinear manner and constructs the optimum separating 
TABLE 3: Testing performance of the proposed identifier at $\mathrm{SNR}=3 \mathrm{~dB}$.

\begin{tabular}{l|l|l|l|l|l|l|l|l|l}
\hline & $\mathrm{P}_{1}$ & $\mathrm{P}_{2}$ & $\mathrm{P}_{3}$ & $\mathrm{P}_{4}$ & $\mathrm{P}_{5}$ & $\mathrm{P}_{6}$ & $\mathrm{P}_{7}$ & $\mathrm{P}_{8}$ & $\mathrm{P}_{9}$ \\
\hline $\mathrm{P}_{1}$ & 97 & & & & & & & & \\
\hline $\mathrm{P}_{2}$ & & 92 & & & & & & & \\
\hline $\mathrm{P}_{3}$ & & & 99 & & & & & & \\
\hline $\mathrm{P}_{4}$ & & & & 95 & & & & & \\
\hline $\mathrm{P}_{5}$ & & & & & 93 & & & & \\
\hline $\mathrm{P}_{6}$ & & & & & & 91 & & & \\
\hline $\mathrm{P}_{7}$ & & & & & & & 92 & & \\
\hline $\mathrm{P}_{8}$ & & & & & & & & 91 & \\
\hline $\mathrm{P}_{9}$ & & & & & & & & & 92 \\
\hline
\end{tabular}

TABle 4: Performances of the proposed identifier in different SNRs without optimization (\%).

\begin{tabular}{ccc}
\hline SNR & Training & Testing \\
\hline-3 & 86.14 & 85.52 \\
0 & 93.26 & 91.45 \\
3 & 94.42 & 93.56 \\
6 & 96.75 & 96.54 \\
9 & 97.85 & 97.56 \\
18 & 98.74 & 98.50 \\
\hline
\end{tabular}

TABle 5: Performances of the identifier with applying of GA.

\begin{tabular}{ccc}
\hline SNR $(\mathrm{dB})$ & Training & Testing \\
\hline-3 & 92.74 & 92.26 \\
0 & 94.92 & 93.64 \\
3 & 96.84 & 96.78 \\
6 & 98.95 & 98.75 \\
9 & 99.14 & 99.12 \\
18 & 99.58 & 99.36 \\
\hline
\end{tabular}

TABLE 6: Optimum parameters of SVMs.

\begin{tabular}{crc}
\hline SVM's number & $C$ & $\sigma$ \\
\hline SVM1 & 2 & 1.2 \\
SVM2 & 10 & 1.4 \\
SVM3 & 14 & 0.9 \\
SVM4 & 18 & 1 \\
SVM5 & 42 & 0.9 \\
SVM6 & 26 & 1.1 \\
SVM7 & 16 & 0.9 \\
SVM8 & 22 & 0.7 \\
\hline
\end{tabular}

hyperplane in the space to realize signal recognition. This classifier avoids the overfitting and local minimum. It shows great generalization ability for identifying the considered digital signal types. The proposed identifier has a success rate of around $92 \%$ at $\mathrm{SNR}=-3 \mathrm{~dB}$. The performance of the identifier is higher than $98 \%$ for SNR $>6 \mathrm{~dB}$. These performances are achieved with few samples.

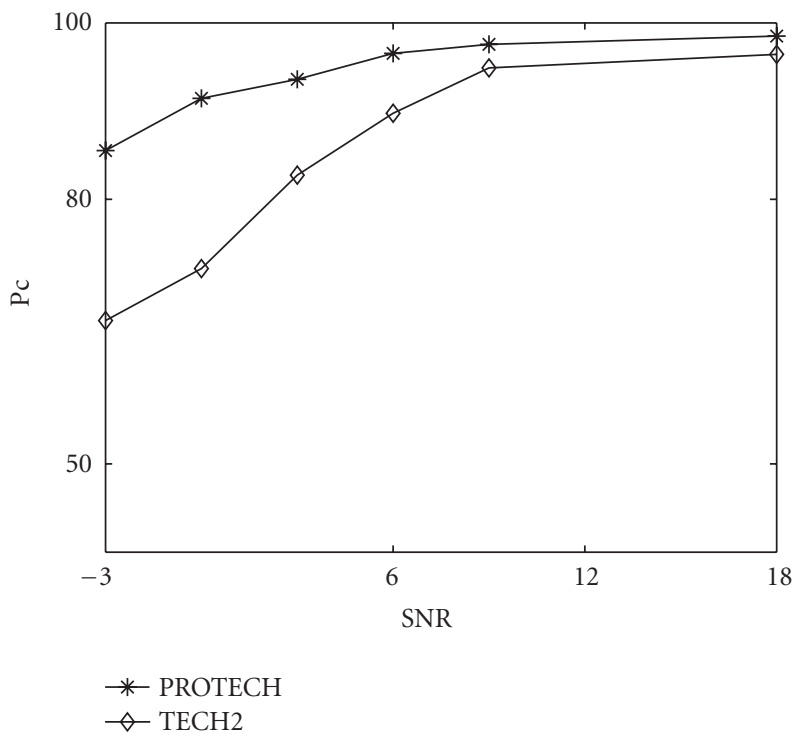

FIGURE 3: Comparison between the performances of PROTECH and TECH2.

\section{CONCLUSIONS}

Automatic digital-signal-type identification has seen increasing demand in different applications. Most of the proposed techniques can only identify low orders of digital signals. They usually require high levels of SNR for identification of the considered digital signals. These problems are mainly due to two facts: the features and the classifier. In this paper, we have used a selected combination of the higher-order moments and the higher-order cumulants up to eighth as the effective features for representation of the digital signal types. These features are selected based on try and error. As the classifier, we have proposed a hierarchical multiclass classifier based on SVMs. This classifier has a simple structure and high generalization ability. By using the mentioned features and the classifier, we have presented a highly efficient identifier. This identifier is able to recognize different types of a digital signal and has a high performance at very low levels of SNR. Optimization of the structure of the classifier improves success rate of the identifier. Therefore, we have used 
TABLE 7: Testing performance of the optimized identifier at SNR $=3 \mathrm{~dB}$.

\begin{tabular}{l|c|c|c|c|c|c|c|c|c}
\hline & $\mathrm{P}_{1}$ & $\mathrm{P}_{2}$ & $\mathrm{P}_{3}$ & $\mathrm{P}_{4}$ & $\mathrm{P}_{5}$ & $\mathrm{P}_{6}$ & $\mathrm{P}_{7}$ & $\mathrm{P}_{8}$ & $\mathrm{P}_{9}$ \\
\hline $\mathrm{P}_{1}$ & 98 & & & & & & & & \\
\hline $\mathrm{P}_{2}$ & & 98 & & & & & & & \\
\hline $\mathrm{P}_{3}$ & & & 100 & & & & & & \\
\hline $\mathrm{P}_{4}$ & & & & 100 & & & & & \\
\hline $\mathrm{P}_{5}$ & & & & & 97 & & & & \\
\hline $\mathrm{P}_{6}$ & & & & & & 96 & & & \\
\hline $\mathrm{P}_{7}$ & & & & & & & 96 & & \\
\hline $\mathrm{P}_{8}$ & & & & & & & & 94 & \\
\hline $\mathrm{P}_{9}$ & & & & & & & & & 92 \\
\hline
\end{tabular}

TABLE 8: Training performance of the optimized identifier at SNR $=40 \mathrm{~dB}$.

\begin{tabular}{l|l|l|l|l|l|l|l|l|l}
\hline & $\mathrm{P}_{1}$ & $\mathrm{P}_{2}$ & $\mathrm{P}_{3}$ & $\mathrm{P}_{4}$ & $\mathrm{P}_{5}$ & $\mathrm{P}_{6}$ & $\mathrm{P}_{7}$ & $\mathrm{P}_{8}$ & $\mathrm{P}_{9}$ \\
\hline $\mathrm{P}_{1}$ & 100 & & & & & & & & \\
\hline $\mathrm{P}_{2}$ & & 100 & & & & & & & \\
\hline $\mathrm{P}_{3}$ & & & 100 & & & & & & \\
\hline $\mathrm{P}_{4}$ & & & & 99.8 & & & & & \\
\hline $\mathrm{P}_{5}$ & & & & & 100 & & & & \\
\hline $\mathrm{P}_{6}$ & & & & & & 100 & & & \\
\hline $\mathrm{P}_{7}$ & & & & & & & 99.6 & & \\
\hline $\mathrm{P}_{8}$ & & & & & & & & 100 & \\
\hline $\mathrm{P}_{9}$ & & & & & & & & & 99.6 \\
\hline
\end{tabular}

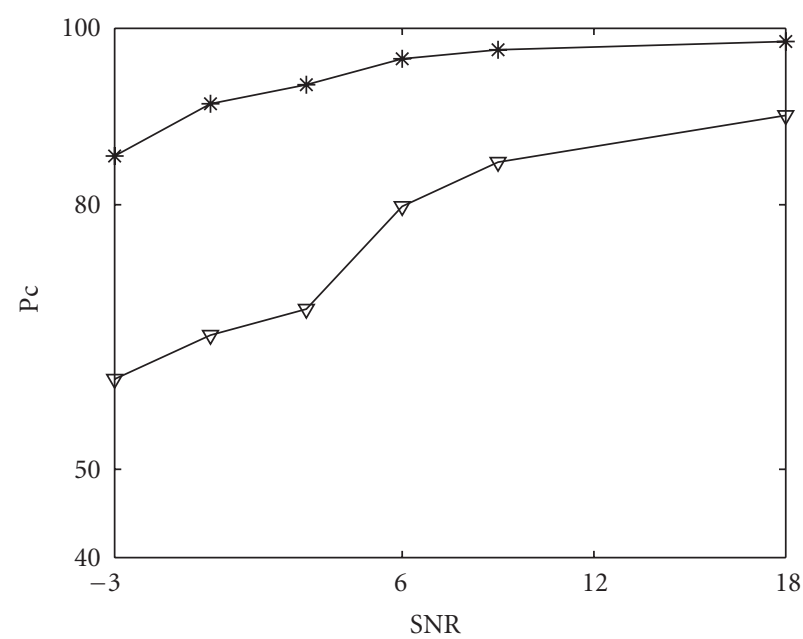

* PROTECH

$\nabla$ TECH3

FIgURE 4: Comparison between the performances of PROTECH and TECH3.

a genetic algorithm as an optimizer in order to achieve the optimum structure of the classifier. This work improves efficiently the performance of the identifier, especially at very low SNRs. For future works, we can use another genetic algorithm and compare the respective results with the results

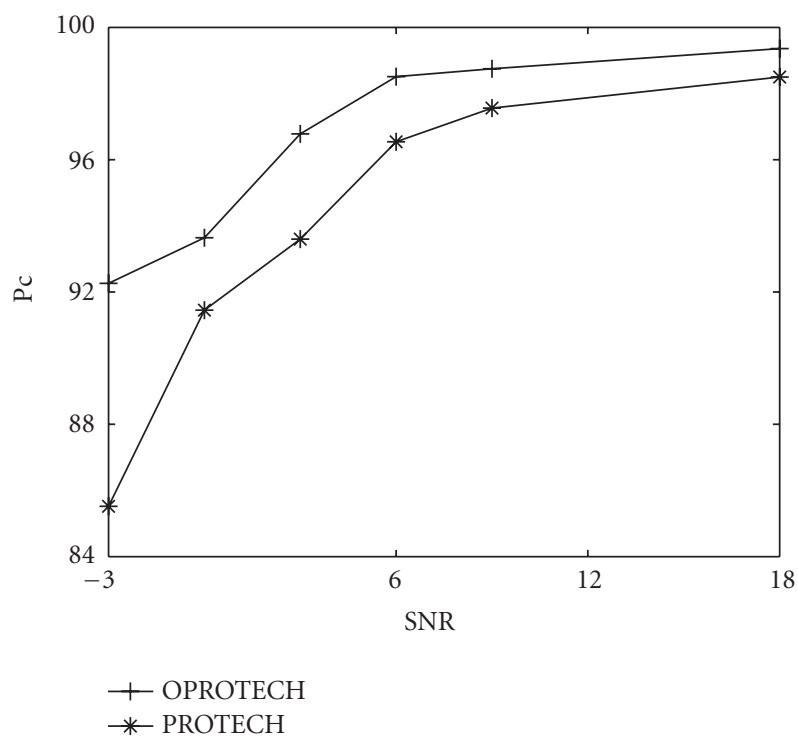

FIgURE 5: Comparison between the performances of the nonoptimized technique (PROTECH) and optimized technique (OPROTECH).

presented in this paper. We can select the proper features introduced by others and use them together with the features that are proposed in this paper in order to have suitable features set for identification of the different types of a digital signal. In this paper, we have used the genetic algorithm 
for optimization of the structure of the classifier. For future works, we can apply the genetic algorithm both for the features subset selection and the optimization of the structure of the classifier.

\section{REFERENCES}

[1] A. Iversen, "The use of artificial neural networks for automatic modulation recognition," Tech. Rep. HW-MACS-TR0009, Heriot-Watt University, Edinburgh, Scotland, December 2003.

[2] W. Wei and J. M. Mendel, "Maximum-likelihood classification for digital amplitude-phase modulations," IEEE Transactions on Communications, vol. 48, no. 2, pp. 189-193, 2000.

[3] P. Panagiotou, A. Anastasopoulos, and A. Polydoros, "Likelihood ratio tests for modulation classification," in Proceedings of the 21st Century Military Communications Conference (MILCOM '00), vol. 2, pp. 670-674, Los Angeles, Calif, USA, October 2000.

[4] A. Swami and B. M. Sadler, "Hierarchical digital modulation classification using cumulants," IEEE Transactions on Communications, vol. 48, no. 3, pp. 416-429, 2000.

[5] M. L. D. Wong and A. K. Nandi, "Automatic digital modulation recognition using artificial neural network and genetic algorithm," Signal Processing, vol. 84, no. 2, pp. 351-365, 2004.

[6] B. G. Mobasseri, "Digital modulation classification using constellation shape," Signal Processing, vol. 80, no. 2, pp. 251-277, 2000.

[7] E. E. Azzouz and A. K. Nandi, "Automatic identification of digital modulation types," Signal Processing, vol. 47, no. 1, pp. 5569, 1995.

[8] J. Lopatka and M. Pedzisz, "Automatic modulation classification using statistical moments and a fuzzy classifier," in Proceedings of the 5th International Conference on Signal Processing (ICSP '00), vol. 3, pp. 1500-1506, Beijing, China, August 2000.

[9] N. Ghani and R. Lamontagne, "Neural networks applied to the classification of spectral features for automatic modulation recognition," in Proceedings of IEEE Military Communications Conference (MILCOM '93), vol. 1, pp. 111-115, Boston, Mass, USA, October 1993.

[10] A. K. Nandi and E. E. Azzouz, "Algorithms for automatic modulation recognition of communication signals," IEEE Transactions on Communications, vol. 46, no. 4, pp. 431-436, 1998.

[11] C. Louis and P. Sehier, "Automatic modulation recognition with a hierarchical neural network," in Proceedings of IEEE Military Communications Conference (MILCOM '94), vol. 3, pp. 713-717, Fort Monmouth, NJ, USA, October 1994.

[12] L. Mingquan, X. Xianci, and L. Leming, "Cyclic spectral features based modulation recognition," in Proceedings of International Conference on Communication Technology (ICCT '96), vol. 2, pp. 792-795, Beijing, China, May 1996.

[13] E. Avci, D. Hanbay, and A. Varol, "An expert discrete wavelet adaptive network based fuzzy inference system for digital modulation recognition," Expert Systems with Applications, vol. 33, no. 3, pp. 582-589, 2007.

[14] A. Abrahamzadeh and S. A. Seyedin, "Automatic modulation type identification using WPA and SVM," International Journal of Tomography \& Statistics, vol. 4, pp. 17-28, 2006.

[15] Z. Wu, X. Wang, Z. Gao, and G. Ren, "Automatic digital modulation recognition based on support vector machines," in Proceedings of International Conference on Neural Networks and Brain (ICNNB '05), vol. 2, pp. 1025-1028, Beijing, China, October 2005.
[16] H. Mustafa and M. Doroslovački, "Digital modulation recognition using support vector machine classifier," in Proceedings of the 38th Asilomar Conference on Signals, Systems and Computers, vol. 2, pp. 2238-2242, Pacific Grove, Calif, USA, November 2004.

[17] H. Gang, L. Jiandong, and L. Donghua, "Study of modulation recognition based on HOCs and SVM," in Proceedings of the 59th IEEE Vehicular Technology Conference (VTC '04), vol. 2, pp. 898-902, Milan, Italy, May 2004.

[18] I. El-Naqa, Y. Yang, M. N. Wernick, N. P. Galatsanos, and R. M. Nishikawa, "A support vector machine approach for detection of microcalcifications," IEEE Transactions on Medical Imaging, vol. 21, no. 12, pp. 1552-1563, 2002.

[19] M. N. Gürcan, Y. Yardimci, A. E. Çetin, and R. Ansari, "Detection of microcalcifications in mammograms using higher order statistics," IEEE Signal Processing Letters, vol. 4, no. 8, pp. 211-216, 1997.

[20] P. McCullagh, Tensor Methods in Statistics, Chapman \& Hall, London, UK, 1987.

[21] C. Cortes and V. Vapnic, "Support vector network," Machine Learning, vol. 20, no. 3, pp. 273-297, 1995.

[22] B. Schölkopf, C. Burges, and V. Vapnik, "Extracting support data for a given task," in Proceedings of the 1st International Conference on Knowledge Discovery and Data Mining (KDD '95), pp. 252-257, Montreal, Canada, August 1995.

[23] O. Chapelle, V. Vapnik, O. Bousquet, and S. Mukherjee, "Choosing multiple parameters for support vector machines," Machine Learning, vol. 46, no. 1-3, pp. 131-159, 2002.

[24] Z. Michalewicz, Genetic Algorithms+Data Structures=Evolution Programs, Springer, New York, NY, USA, 3rd edition, 1999.

Ataollah Abrahamzadeh was born in Babolsar at the north of Iran. He received his Ph.D. degree in electrical engineering from Ferdowsi University of Mashad, Mashad, Iran, in 2006. Now he is an Assistant Professor in the Faculty of Electrical and Computer Engineering at Noushirvani Institute of Technology. His current scientific interests are in general area of signal processing, biomedical engineering, and artificial intelligence.

Seyed Alireza Seyedin was born in Iran. He received the B.S. degree in electronics engineering from Isfahan University of Technology, Isfahan, Iran, in 1986 and the M.E. degree in control engineering from Roorkee University, Roorkee, India, in 1992 and the Ph.D. degree in electrical engineering from the University of New South Wales, Sydney, Australia, in 1996. Since 1996, he has been with the Faculty of Engineering, Ferdowsi

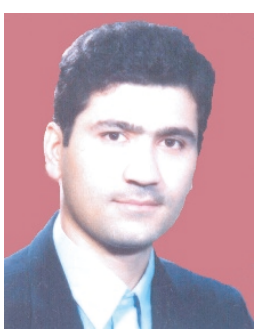
University of Mashhad, Mashhad, Iran, as an Assistant Professor. He was the Head of the Department of Electrical Engineering for the period 1998-2000. He is a Member of Scientific Committee of the Iranian Conference on Machine Vision and Image Processing. His current research interests include image analysis, digital signal processing, robotics, machine vision, and pattern recognition. 
Mehdi Dehghan received his B.S. degree in computer engineering from Iran University of Science and Technology (IUST), Tehran, Iran, in 1992, and his M.S. and Ph.D. degrees from Amirkabir University of Technology (AUT), Tehran, Iran, in 1995, and 2001, respectively. He is an Assistant Professor of Computer engineering and information technology at Amirkabir University of Technology (AUT). Prior to joining AUT in

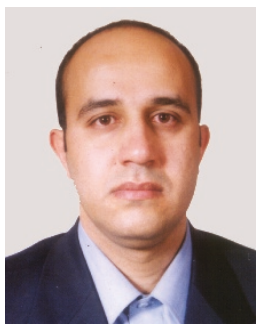

2004, he was a Research Scientist at Iran Telecommunication Research Center (ITRC) working in the area of quality-of-service provisioning and network management. His research interests are in wireless networks, pattern recognition, fault-tolerant computing, and distributed systems. 